\title{
An Analysis of Factors Influencing the Self-Rated Health of Elderly Chinese People
}

\author{
Ping Gao, Handong Li \\ Department of Management Science and Engineering, School of Government, Beijing Normal University, \\ Beijing, China \\ Email: gaoping4069111@126.com, lhd@bnu.edu.cn
}

Received 17 March 2015

\begin{abstract}
This study used the data from the Chinese Urban and Rural Elderly Population Survey to analyze the correlations among the self-rated health of elderly Chinese people, demographic factors and chronic diseases. The results revealed that in comparing younger elderly individuals vs older elderly individuals, the urban elderly vs rural elderly, elderly man vs elderly women, and the elderly with fewer chronic diseases vs the elderly with more chronic diseases, the former's self-rated health tended to be good, while the latter tended to be bad. In addition, over time, elderly individuals' self-rated health tended to improve. This paper further studied high blood pressure, heart disease, arthritis, neck and lumbar disease, and chronic bronchitis and the impact of these five common chronic diseases on self-rated health. The results showed that, among these five types of chronic diseases, the impacts of heart disease and chronic bronchitis were greater than those of the other three chronic diseases.
\end{abstract}

\section{Keywords}

Chronic Disease, Self-Rated Health, Polytomous Logistic Regression for Ordinal Response

\section{Introduction}

Self-rated health is a comprehensive assessment based on an elderly person's subjective feelings. It can reflect not only population differences but also the common factors affecting the health of the elderly. Among the researchers investigating the factors influencing self-rated health, Tian Feng and Zhen Zhengzheng (2004) revealed that an elderly person's sex, age, education and other personal characteristic could affect self-rated health, but these factors were not more significant than health-related factors [1]. Sun Yuhua (2013) reported that self-rated health was not only relative to sex, age and other demographic factors but also to chronic diseases [2]. In the area of the relationships between health conditions and chronic diseases, Fang Xianghua et al. (2003) found that elderly people's self-rated health was relative to the rates of common geriatric chronic diseases [3]. Li Cuixia et al. (2012) statistically analyzed common chronic diseases and self-rated health conditions, and they performed unconditioned logistic regression of influencing factors to analyze the relationships between the self-rated health and chronic diseases in Nanning [4]. Zhang Fengmei and Xu Hengjian (2008) undertook single 
factor and multiple factor analyses to assess the relationships between self-rated health and chronic diseases, and they noted that the self-rated health of elderly individuals was closely linked to the number and type of chronic diseases [5].

This study first analyzed the impacts of demographic characteristics on the elderly's self-rated health and then further studied the relationships between self-rated health and common chronic diseases.

\section{Data and Method}

\subsection{Data and Processing}

The data for this study were obtained from the 2006 and 2010 Chinese Urban and Rural Elderly Population Surveys, conducted by the China Research Center on Aging of the National Committee on Aging. These data cover the following 20 provinces, municipalities and autonomous regions in China: North China-Beijing, Hebei, and Shanxi; Northeast China—Liaoning and Heilongjiang; East China—Shanghai, Jiangsu, Zhejiang, Anhui, Fujian, and Shandong; Mid-South China-Henan, Hubei, Hunan, Guangdong, and Guangxi; Southwest China-Sichuan and Yunnan; and Northwest China-Shanxi and Xinjiang.

The original questionnaire's self-rated health had five possible levels: very bad, bad, general, good, and very good; this paper combined them into three levels, that is, very bad and bad were combined into bad, general remained general, and good and very good were combined into good, so the elderly's self-rated health finally had three levels: bad, general and good. Because our dependent variable was an orderly ternary variable, we used the method of polytomous logistic regression for ordinal responses to analyze.

In this study, the data processing was based on the Ordinal Regression function in SPSS software.

\subsection{The Influencing Factors and Assignment}

The paper studied the demographic factors and chronic diseases that affected the elderly's self-rated health. The demographic factors included age, sex and census registry. The chronic disease factors included five chronic diseases: high blood pressure, heart disease, arthritis, neck and lumbar disease, and chronic bronchitis. The factors' assignments were as follows Table 1.

\section{Model Estimation Results and Analysis}

\subsection{Ordinal Logistic Regression of Chronic Diseases}

Placing age, sex, census registry and whether one is suffering from chronic diseases into the Ordinal Logistic Regression model, the model fitting information was as follows.

From Table 2 we can see that the -2 logarithm likelihood value declined sharply from 5759.150 to 2583.432 after the mode with the four factors included. The Sig. is $0.000<0.001$, and this value revealed that the final model was highly statistically significant. In the final model, Pearson's chi-square and deviance were 0.006 and 0.001 , respectively; both values were small, so the goodness of fit of the model was very good.

\section{Table 1. Factors' assignment.}

\begin{tabular}{|c|c|c|}
\hline Level one variable & The secondary variables & Assignment \\
\hline Dependent variable & Self-rated health & Bad is 1 ; general is 2 ; good is 3 . \\
\hline \multirow[t]{3}{*}{ Demographic factors } & Sex & Male is 1 ; female is 2 . \\
\hline & Census registry & Rural is 1 ; urban is 2 . \\
\hline & Age & Continuous variable \\
\hline \multirow[t]{6}{*}{ Chronic disease factors } & Whether one is suffering from chronic diseases & No is 0 ; yes is 1 . \\
\hline & Whether one is suffering from high blood pressure & No is 0 ; yes is 1 . \\
\hline & Whether one is suffering from heart disease & No is 0 ; yes is 1 . \\
\hline & Whether one is suffering from arthritis & No is 0 ; yes is 1 . \\
\hline & Whether one is suffer from neck lumbar disease & No is 0 ; yes is 1 . \\
\hline & Whether one is suffering from chronic bronchitis & No is 0 ; yes is 1 . \\
\hline
\end{tabular}


Table 2. Model fitting information (2006).

\begin{tabular}{ccccc}
\hline Model & -2 logarithm likelihood values & Chi-square & Df & Sig. \\
\hline Only intercept & 5759.150 & & & \\
Final & 2583.432 & 3175.718 & 4 & 0.000 \\
\hline
\end{tabular}

In this final model, the dependent variable was the elderly's self-rated health, and the independent variables were age, sex, census registry and whether one was suffering from chronic diseases. The parameter estimation results are presented in Table 3 (During the data processing, the last classification was for reference, so all parameter values of the variables' last classification were 0 , and they are omitted from the table).

Based on the parameter estimation results and accumulative Logit fomula:

$$
\log i t P_{j}=\log i t[P(y>j \mid x)]=\ln \frac{P(y>j \mid x)}{1-P(y>j \mid x)}=-\alpha_{j}+\sum \beta_{i} x_{i}
$$

the data in 2006 can be fitted into the following accumulative Logit model:

$\log i t[P(y>j \mid x)]=-\alpha_{j}-0.043 \times$ Age $+0.297 \times[$ Sex $=1]-0.526 \times[$ Census registry $=1]+1.605 \times$

[Chronic disease $=0$ ]

where $j=1,2 ; \quad \alpha_{1}=-4.064, \quad \alpha_{2}=-1.439$.

In the same manner, we can use the data in 2010 to fit the following accumulative Logit model:

$\log i t[P(y>j \mid x)]=-\alpha_{j}-0.049 \times$ Age $+0.248 \times[$ Sex $=1]-0.588 \times[$ Census registry $=1]+1.454 \times$

[Chronic disease $=0$ ]

where $j=1,2 ; \quad \alpha_{1}=-4.644, \alpha_{2}=-2.008$.

On the right side of the formula, there are two intercept values, which are used to calculate the probability of the bad level and the general level.

From the equation, we can see that this accumulative Logit model is a probability formula regarding the self-rated health level. Thus, if we want to calculate one's self-rated health level, we must obtain different levels' probabilities first and determine which level's probability is the greatest; then, the corresponding level is an individual's self-rated health level. The calculation of different levels' probabilities is as follows.

Based on the cumulative Logit model we can derive that:

$$
P(y>j \mid x)=\exp \left(-\alpha_{j}+\sum \beta_{i} x_{i}\right) /\left[1+\exp \left(-\alpha_{j}+\sum \beta_{i} x_{i}\right)\right]
$$

Because $P(y>j \mid x)=1-P(y \leq j \mid x)$, so

$$
P(y \leq j \mid x)=1 /\left[1+\exp \left(-\alpha_{j}+\sum \beta_{i} x_{i}\right)\right]
$$

In addition,

$$
P(y \leq j \mid x)=P(y=1 \mid x)+\cdots+P(y=j \mid x)
$$

Then, we can obtain different levels’ probability by calculation using the formula (when $j=1,2$, and 3 ):

$$
\begin{gathered}
P(y=1 \mid x)=1 /\left[1+\exp \left(-\alpha_{1}+\sum \beta_{i} x_{i}\right)\right] \\
P(y=2 \mid x)=1 /\left[1+\exp \left(-\alpha_{2}+\sum \beta_{i} x_{i}\right)\right]-1 /\left[1+\exp \left(-\alpha_{1}+\sum \beta_{i} x_{i}\right)\right] \\
P(y=3 \mid x)=1-1 /\left[1+\exp \left(-\alpha_{2}+\sum \beta_{i} x_{i}\right)\right]
\end{gathered}
$$

We can see from the accumulative Logit models in 2006 and 2010, the coefficient of age is negative, that is, the greater the age is, the smaller the $\sum \beta_{i} x_{i}$ is, thus the greater the $P(y=1 \mid x)$ is, the smaller the $P(y=3 \mid x)$ is, that is, the older the person is, the greater the probability of bad health is, while the smaller the probability of good health is. The coefficient of census registry is negative, that is, when comparing the rural elderly to the urban elderly, the former's $\sum \beta_{i} x_{i}$ is smaller, so the greater the $P(y=1 \mid x)$ is, the smaller the $P(y=3 \mid x)$ is, that is, the rural elderly's probability of having bad health is greater than that of the urban elderly's, while the probability of good health is smaller than that of the urban elderly's. The coefficient of sex is positive, that is, in comparing the male elderly to the female elderly, the former's $\sum \beta_{i} x_{i}$ is greater, so the smaller the $P(y=1 \mid x)$ is, the greater the $P(y=3 \mid x)$ is, that is, the male elderly's probability of bad health is smaller than that of the 
Table 3. Parameter estimation results (2006).

\begin{tabular}{|c|c|c|c|c|c|c|c|c|}
\hline & \multirow{2}{*}{ Variable } & \multirow{2}{*}{ Estimation } & \multirow{2}{*}{ SE } & \multirow{2}{*}{ Wald } & \multirow{2}{*}{ Df } & \multirow{2}{*}{ Sig. } & \multicolumn{2}{|c|}{ 95\% confidence interval } \\
\hline & & & & & & & Lower & Upper \\
\hline \multirow[t]{3}{*}{ Intercept } & [Self-rated health $=1]$ & -4.064 & 0.147 & 764.969 & 1 & 0.000 & -4.352 & -3.776 \\
\hline & [Self-rated health $=2$ ] & -1.439 & 0.144 & 99.690 & 1 & 0.000 & -1.721 & -1.156 \\
\hline & Age & -0.043 & 0.002 & 456.537 & 1 & 0.000 & -0.047 & -0.039 \\
\hline \multirow{3}{*}{$\begin{array}{l}\text { Independent } \\
\text { variable }\end{array}$} & {$[$ Sex $=1]$} & 0.297 & 0.028 & 113.277 & 1 & 0.000 & 0.242 & 0.352 \\
\hline & [Census registry = 1] & -0.526 & 0.028 & 344.887 & 1 & 0.000 & -0.581 & -0.470 \\
\hline & [Chronic disease $=0$ ] & 1.605 & 0.034 & 2165.112 & 1 & 0.000 & 1.537 & 1.672 \\
\hline
\end{tabular}

female elderly's, while the probability of good health is greater than that of the female elderly's. The coefficient of chronic disease is positive, so it has similar properties to sex, so in comparing the elderly not suffering from chronic diseases to the elderly suffering from chronic diseases, the former's probability of bad health is smaller than that of the latter, while the probability of good health is greater than that of the latter.

In conclusion, in comparing the younger elderly vs older elderly, the urban elderly vs rural elderly, the male elderly vs female elderly, and the elderly not suffering from chronic diseases vs the elderly suffering from chronic diseases, the former's self-rated health tended to be good, while the latter's health tended to be bad.

In addition, for the elderly people of the same age, in the same census registry, with the same sex, and with the same chronic conditions, the $P(y=1 \mid x)$ in 2006 was greater than that in 2010, while the $P(y=3 \mid x)$ in 2006 was smaller than that in 2010; that is, as time passed, the elderly's self-rated health tended to be better.

\subsection{Ordinal Logistic Regression of Common Chronic Diseases}

Placing age, sex, census registry and whether one is suffering from high blood pressure, heart disease, arthritis, neck and lumbar disease, or chronic bronchitis into an Ordinal Logistic Regression model, the model fitting information was as follows.

From the Table 4 we can see that, the -2 logarithm likelihood value declined sharply from 13148.407 to 10501.371 after the model had the eight factors in it. The Sig. is $0.000<0.001$, and this value revealed that the final model was highly statistically significant. In the final model, the Pearson's chi-square and Deviance were 0.049 and 0.000 , respectively; both values were small, so the goodness of fit of the model was good.

In this final model, the dependent variable was the elderly's self-rated health, and the independent variables were age, sex, census registry and whether one is suffering from high blood pressure, heart disease, arthritis, neck and lumbar disease, or chronic bronchitis. The parameter estimation results followed in Table 5. (During the data processing, the last classification was for reference, so all of the parameter values of the variables' last classification were 0 ; they were omitted from the table).

Based on the parameter estimation results and accumulative Logit fomula, the data from 2006 can be fitted the following accumulative Logit model:

$\operatorname{logit}[P(y>j \mid x)]=-\alpha_{j}-0.045 \times$ Age $+0.266 \times[$ Sex $=1]-0.580 \times[$ Census registry $=1]+0.486 \times[$ High blood pressure $=0]+0.794 \times[$ Heart disease $=0]+0.464 \times[$ Arthritis $=0]+0.392 \times[$ Neck lumbar disease $=0]+$ $0.670 \times$ [Chronic bronchitis $=0$ ]

where $j=1,2 ; \quad \alpha_{1}=-2.385, \quad \alpha_{2}=-0.185$.

In the same manner, we can use the data from 2010 to fit the following accumulative Logit model:

$\operatorname{logit}[P(y>j \mid x)]=-\alpha_{j}-0.051 \times$ Age $+0.221 \times[$ Sex $=1]-0.635 \times[$ Census registry $=1]+0.370 \times[$ High blood pressure $=0]+0.713 \times[$ Heart disease $=0]+0.383 \times[$ Arthritis $=0]+0.431 \times[$ Neck lumbar disease $=0]$ $+0.563 \times[$ Chronic bronchitis $=0$ ]

where $j=1,2 ; \quad \alpha_{1}=-3.185, \quad \alpha_{2}=-0.585$.

From the cumulative Logit model in 2006 and 2010, we find that the coefficient of age is negative, the coefficient of census registry is negative, the coefficient of sex is positive, and the coefficients of the five 
Table 4. Model fitting information (2006).

\begin{tabular}{ccccc}
\hline Model & -2 logarithm likelihood values & Chi-square & Df & Sig. \\
\hline Only intercept & 13148.407 & & \\
Final & 10501.371 & 2647.036 & 8 & 0.000 \\
\hline
\end{tabular}

Table 5. Parameter estimation results (2006).

\begin{tabular}{|c|c|c|c|c|c|c|c|c|}
\hline & \multirow{2}{*}{ Variable } & \multirow{2}{*}{ Estimation } & \multirow{2}{*}{ SE } & \multirow{2}{*}{ Wald } & \multirow{2}{*}{ Df } & \multirow{2}{*}{ Sig. } & \multicolumn{2}{|c|}{ 95\% confidence interval } \\
\hline & & & & & & & Lower & Upper \\
\hline \multirow[t]{3}{*}{ Intercept } & [Self-rated health = 1] & -2.385 & 0.156 & 233.620 & 1 & 0.000 & -2.691 & -2.079 \\
\hline & [Self-rated health = 2] & 0.185 & 0.155 & 1.425 & 1 & 0.233 & -0.119 & 0.488 \\
\hline & Age & -0.045 & 0.002 & 501.005 & 1 & 0.000 & -0.049 & -0.041 \\
\hline \multirow{6}{*}{$\begin{array}{l}\text { Independent } \\
\text { variable }\end{array}$} & {$[$ Sex $=1]$} & 0.266 & 0.028 & 90.422 & 1 & 0.000 & 0.211 & 0.321 \\
\hline & [Census registry = 1] & -0.580 & 0.029 & 397.418 & 1 & 0.000 & -0.637 & -0.523 \\
\hline & [High blood pressure = 0] & 0.486 & 0.032 & 233.380 & 1 & 0.000 & 0.424 & 0.548 \\
\hline & {$[$ Heart disease $=0]$} & 0.794 & 0.037 & 458.272 & 1 & 0.000 & 0.721 & 0.867 \\
\hline & [Arthritis = 0] & 0.464 & 0.034 & 188.822 & 1 & 0.000 & 0.398 & 0.530 \\
\hline & {$[$ Neck lumbar disease $=0]$} & 0.392 & 0.041 & 90.090 & 1 & 0.000 & 0.311 & 0.472 \\
\hline
\end{tabular}

chronic diseases are positive; these results coincide with the former analysis. Because the coefficients of the five chronic diseases are positive, the smaller the number of an elderly person's chronic diseases is, the greater the $\sum \beta_{i} x_{i}$ is, and so the smaller the $P(y=1 \mid x)$ is, the greater the $P(y=3 \mid x)$ is, that is, the smaller the number of chronic diseases is, the greater the probability is of good health, while the smaller the probability is of bad health. In addition, even if the numbers of chronic diseases of two people are the same, if their diseases are not the same, their self-rated health is different because the diseases' coefficients are different.

However, in both 2006 and 2010, the coefficient of heart disease is the greatest, followed by the coefficient of chronic bronchitis, that is, heart disease and chronic bronchitis have the greater impacts on self-rated health than the other three diseases.

In conclusion, in comparing younger elderly people vs older elderly people, the urban elderly vs rural elderly, elderly men vs elderly women, and the elderly with fewer chronic diseases vs the elderly with more chronic diseases, the former's self-rated health tended to be good, while the latter's tended to be bad. Even if the numbers of chronic diseases of two people were the same, the self-rated health was different because the coefficients of the chronic diseases were different.

\section{Conclusion}

This study used the data from the Chinese Urban and Rural Elderly Population Survey and use Ordinal Logistic Regression to analyze the correlations among Chinese elderly peoples' self-rated health, demographic factors and chronic diseases. The results revealed that in comparing younger elderly people vs older elderly people, the urban elderly vs rural elderly, elderly men vs elderly women, and the elderly with fewer chronic diseases vs the elderly with more chronic diseases, the former's self-rated health tended to be good, while the latter's tended to be bad. In addition, as time passed, the elderly's self-rated health tended to improve. This paper further studied the impacts of five common chronic diseases on self-rated health as follow: high blood pressure, heart disease, arthritis, neck and lumbar disease, and chronic bronchitis. The results showed that, among the five types of chronic diseases, the impacts of heart disease and chronic bronchitis were greater than those of the other three chronic diseases' impact. In 2006, the five diseases' impacts on self-rated health were as follows (from largest to smallest): heart disease, chronic bronchitis, high blood pressure, arthritis, and neck and lumbar disease; in 2010, the five diseases' impacts on self-rated health were as follows (from largest to smallest): heart disease, chronic bronchitis, neck and lumbar disease, arthritis, high blood pressure. 


\section{References}

[1] Tian, F. and Zhen, Z.Z. (2004) The Change of Elderly’s Self-Rated Health and Its Influencing Factors. Population Science of China, S1, 63-69.

[2] Sun, Y.H. (2013) The Status and Influencing Factors of Self-Rated Health of the Elderly. Master Thesis, Jilin University, Jilin.

[3] Fang, X.H., Meng, C., Liu, X.H., et al. (2003) Self-Rated Health and the Elderly's Health Research. Chinese Journal of Epidemiology, 24, 184-188.

[4] Li, C.X., Pan, Z.M., Zeng, X.Y. and Xiao, D.Q. (2012) The Relationships between Chronic Diseases Distribution and Self-Rated Health of Nanning's Community Elderly. Chinese Journal of Gerontology, 32, 3748-3750.

[5] Zhang, F.M. and Xu, H.J. (2008) The Relationship Research of Self-Rated Health and the Elderly's Chronic Disease. Chinese Journal of Gerontology, 23, 2353-2355. 